\section{Anasarca in a 35 year old man- A diagnostic dilemma}

\author{
Okafor UH*, Okorie GO and Ede S
}

Renal Unit, ESUTH Parklane, Enugu, Nigeria

\begin{abstract}
Anasarca is generalized swelling of the body following accumulation of fluid in the extracellular compartments. It may result from multiple aetiology mainly of renal, hepatic or cardiovascular origin.

The aim of this case report is to highlight the challenges encountered in making diagnosis in a patient with anarsaca.

We report a case of a 34 year old transporter who presented with anasarca. He had clinical features and risk factors suggestive of renal, hepatic and cardiovascular disease. However investigations ruled out renal, hepatic or cardiovascular diseases as the aetiology of the anarsaca. The anarsarca was also noted to be unresponsive to diuretics. The diagnosis of the disease causing the anarsaca was therefore a dilemma.
\end{abstract}

\begin{abstract}
More Information
Submitted: 27 June 2019

Approved: 16 July 2019

Published: 17 July 2019

How to cite this article: Okafor UH, Okorie GO, Ede S. Anasarca in a 35 year old man- A diagnostic dilemma. Arch Case Rep. 2019; 3: 014-016. https://doi.org.10.29328/journal.acr.1001014

Copyright: ( 2019 Okafor UH, et al. This is an open access article distributed under the Creative Commons Attribution License, which permits unrestricted use, distribution, and reproduction in any medium, provided the original work is properly cited
\end{abstract}

ISSN: 2637-3793

\section{Introduction}

Anasarca also known as extreme generalized oedema is a generalized body swelling resulting from marked retention of extracellular fluid in the interstitium and the potential spaces. It has been reported to result from imbalance between the oncotic and hydrostatic pressure in both intravascular and extravascular compartment of the extracellular space. Clinical conditions that cause decrease in intravascular oncotic pressure or extravascular/interstitial hydrostatic pressure usually present with various severity of oedema including anarsaca. Also conditions that increase intravascular hydrostatic pressure or extravascular oncotic pressure may present with anarsaca though very rare. Diseases affecting the integrity of the vessels especially the capillaries can also cause anasarca. There may be combination of these various mechanisms in some diseases [1,2].

Clinical conditions associated with anarsaca include acute/chronic kidney diseases including nephrotic syndrome, chronic liver disease, heart failure, septicaemia, malignancies and any other clinical condition presenting with angioedema and/or malnutrition including Beri Beri [1]. Also anarsaca has been reported though rare in these clinical conditions: haemoglobin Barts, acute hepatitis B, acute hepatitis C, anticancer chemotherapy, dihydropyrine calcium chanel blockers usage, fluid therapy, and capillary leak syndrome [3].
The above wide array of aetiology associated with anasarca makes diagnosis a challenge and requires detailed and careful assessment including laboratory and imaging investigations. Thus there may need for multidisciplinary approach in management of anarsaca.

The aim of this case report is to highlight the challenges in making diagnosis of the cause of anarsaca in the 34 years old man.

\section{Report of Index Case}

Mr AC, a 34yr old attendant in a transport company presented with progressive abdominal and leg swelling of 3 months duration. He had dyspnoea on exersion but no orthopnoea, paroxysmal nocturnal dyspnoea, cough, chest pain nor palpitations.

He had anorexia, early satiety, weight loss, nausea and occasionally post prandial non projectile vomiting. He had no fever, night sweat, jaundice, pruritus nor urinary symptoms. However he had febrile illness associated with jaundice 6 months prior to current symptoms but these resolved completely within a week following treatment with herbs.

He was diagnosed of hypertension and was found to be positive for HBsAg during a medical outreach a year prior to presentation but was neither followed up nor on medication. 
There was no past history of diabetes mellitus, sore throat, skin rashes, and sickle cell disease.

He had a significant history of alcohol ingestion (36 units weekly for about 8 years) but neither smoked nor took analgesic regularly. Both parent were hypertensive but no family history of diabetes mellitus, renal or hepatic disease. He resorted to herbs at the onset of this illness but noticed he was deteriorating and thus presented to us at Enugu State University Teaching Hospital Parklane Enugu Nigeria.

Physical examination showed a chronically ill looking young man, in mild respiratory distress, pale, anicteric, acyanotic, no finger clubbing, had facial puffiness, pitting edema of the lower limbs extending to the scrotum and sacrum. The abdomen was markedly distended with ascites demonstrable by fluid thrill. There was no visible abdominal veins and no organs was palpable. His pulse was 70 beats/ minute (NVR), synchronous with other peripheral pulses, no thickened arterial wall, and no locomotor brachialis. The BP was140/80mmHg, JVP was not raised. Apex beat was at 5 th left inter costal space, mid clavicular line, not heaving, only first and second heart sounds were heard, no murmur. The respiratory rate was $26 / \mathrm{min}$, breath sound was vesicular and no crepitations. Nervous system examination showed no abnormality.

An initial assessment of Liver cirrhosis r/o nephrotic syndrome was made. He was admitted and commenced on frusemide $40 \mathrm{mg}$ daily, Tabs spironolactone $25 \mathrm{mg}$ bd, Tabs fesolate $400 \mathrm{mg}$ tds. Investigations results showed: The Liver Function Test showed mildly elevated AST (108.5iu/L) and ALT (55.1iu/L). Other parameters were normal. Gamma glutamyl transferase and alpha feto protein were normal. Total protein $(10.8 \mathrm{~g} / \mathrm{l})$ and albumin $(4.0 \mathrm{~g} / \mathrm{l})$ were normal. The HBsAg was reactive but HCV/HIV were negative, The hepatitis B viral load and antibody were not done.

The serum electrolyte, urea and creatinine were normal, urinalysis was normal, 24 hour urinary protein(101.5mg) and Creatinine $\mathrm{cl}(98 \mathrm{ml} / \mathrm{min})$ were normal, full blood count was normal, ESR was $116 \mathrm{~mm} / 1$ st hour. The lipid profile showed hypercholesterolaemia (total cholesterol $-6.6 \mathrm{mmol}$, HDL - $0.8 \mathrm{mmol} / \mathrm{l}$, LDL - $4.1 \mathrm{mmol}$, VLDL - 1.7mmol/l, triglyceide $-3.7 \mathrm{mmol} / \mathrm{l}$ ).

Abdomino-pelvic ultrasound showed massive ascites and omental cake. Other abdominal viscera were normal. The chest $\mathrm{x}$ ray was normal. ECG showed low limb voltage otherwise normal. Echocardiography showed dilated right atrium, other chambers were normal in size and structure, no intracardiac thrombi, good atrial and ventricular contraction and functions, pericardium was normal. Mild diastolic dysfunction. Mantoux test was negative. Diagnostic abdominal paracentesis showed that the ascitic fluid was serous, exudative, sterile and had scanty lymphocyte.
The problems of the patients were anarsaca, past history of jaundice, hypertension, HBsAg positive, elevated AST/ALT, exudative ascites, omental cake, elevated ESR, low voltage ECG, dilated right atrium and mild diastolic dysfunction.

A definitive diagnosis could not be made and patient did not respond to the above diuretics. Thus the furusemide was later increased to $100 \mathrm{mg}$ bd and spironolactone $100 \mathrm{mg}$ bd, tabs metolazone $5 \mathrm{mg}$ daily, tabs rosuvastatin $10 \mathrm{mg}$ daily, tabs vasoprin $75 \mathrm{mg}$ daily were also added but patient did not respond after two weeks of admission. He was subsequently commenced on anti tuberculous therapy of caps rifampicin $600 \mathrm{mg}$ daily, isoniazid $300 \mathrm{mg}$ daily, ethambutol $800 \mathrm{mg}$ daily, pyrazinamide $1500 \mathrm{mg}$ daily, tabs pyridoxine $25 \mathrm{mg}$.

Patient showed remarkable progressive improvement within a week with regression of oedema, improved appetite and well-being. He was discharged home with the anti $\mathrm{Tb}$ medications 2 weeks later to be followed up in the medical out patient. He has been regular to follow up and adherent to the medications. The oedema has regressed completely and he has resumed work.

\section{Discussion}

It is usual to consider diseases of the liver, kidneys and heart when a patient presents with anarsaca. Thus most investigations are focused on these organs to elucidate the aetiology of anasarca. However causes of anarsaca are not restricted to these three organs as there are other uncommon causes. This index case presented with some remarkable challenges in making diagnosis.

The clinical scenario of the patient suggest he may be having chronic kidney disease considering the multiple risk factors - herbal ingestion, hypertension, hepatitis B infection and the pattern of the anarsaca. However the results of laboratory investigations was not consistent with kidney disease. There was no evidence of injury to the kidney nor impairment in its function as depicted by KDOQI definitionof kidney disease [4].

Index case also presented with past history of jaundice, hepatitis B infection, herbal ingestion which could be hepatotoxic, deranged liver enzymes which is suggestive of liver disease. However the serum protein and albumin was normal, the ascites was exudative and there was no evidence of portal hypertension; thus not consistent with anarsaca resulting from liver disease.

Diseases of the heart is a possibility in this patient in view of the breathlessness initially on exertion and then at rest, history of hypertension, ingestion of herbs which could be cardiotoxic, low voltage ECG in addition to the anarsaca. The absence of tachycardia, pulmonary oedema, hepatomegaly, elevated JVP, $3^{\text {rd }}$ heart sound, and the echocardiographic findings were not consistent with heart disease. 
Other reported causes of anarsaca including malignancy and its treatment, malnutrition, anaphylaxis, myxedema and septicaemia were not evident as the cause of the anasarca in this patient. Other reported but rare causes of anarsaca include pituitary and vascular lymphoma, primary intestinal telangiectasia, Menetrier's disease.

Thus the diagnosis of anarsaca in this patient posed a significant challenge following the exclusion of the above diseases. However in view of the anorexia, unplanned weight loss, elevated ESR, exudative lymphocytic ascitis, omental cake found on abdominal ultrasound and patient living in endemic tuberculosis environment, abdominal tuberculosis was suspected and thus anti tuberculosis therapy was commenced. The patient responded to this therapy with clinical improvement, thus suggestive that tuberculosis is the aetiology of the anarsaca.

Anasarca is a very rare presentation in patients with tuberculosis and there are few case reports. Madhav Bensal et al. [5] reported a case of a 30 year old man with pulmonary tuberculosis who presented with anasarca. The anasarca resolved following treatment of the tuberculousis with anti TB medications. This outcome following anti TB therapy was consistent with the index case and other reports.

Renal amyloidosis resulting from TB has been reported as a cause of nephrotic syndrome with resultant anarsaca $[5,6]$. Anarsaca has also been reported in patients suffering from TB pericarditis $[7,8]$. However the index patient has neither renal nor pericardial disease.

Tuberculosis is a chronic inflammatory disease affecting diffuse organs including the capillaries causing capillary leak syndrome that can lead to anarsaca [9]. This appears to reflect the mechanism of the anasarca in the index patient.
Anasarca which is a common clinical presentation can present challenges as the index case both in diagnosis and management even to very experienced clinicians. We are reporting this case to highlight these challenges and the need for comprehensive and scrupulous assessment of patients presenting with anasarca especially in environment like ours with limited resources and diagnostic tools.

\section{References}

1. de Pietro M. What is anasarca?. Medical News Today. 2019; https:// www.medicalnewstoday.com/articles/320906.php.

2. Klanderman RB, Bosboom JJ, Migdady $\mathrm{Y}$, Veelo DP, Geerts BF, et al. Transfusion-associated circulatory overload-a systematic review of diagnostic biomarkers. Transfusion. 2019; 59: 795-805. PubMed: https://www.ncbi.nlm.nih.gov/pubmed/30488959

3. Sica DA. Calcium Channel Blocker Related Peripheral Edema: Can It Be Resolved? J Clin Hypertens. 2003; 5: 291-295. PubMed: https:// www.ncbi.nlm.nih.gov/pubmed/12939574

4. Chapter1: Definition and classification of CKD. Kidiney Int suppl. 2013; 3: 19 - 62.

5. Dixit R, Gupta R, Dave L, Prasad N, Sharma S. Clinical profile of patients having pulmonary tuberculosis and renal amyloidosis. Lung India. 2009; 26: 41-5. PubMed: https://www.ncbi.nlm.nih.gov/ pubmed/20440393

6. Mandal SK, Sil K, Ganguly J, Chatterjee K, Chatterjee S, et al. Two cases of nephrotic syndrome with different etiologies. Ann Trop Med Public Health. 2013; 6: 105-8.

7. Dayal D, Das A, Pilanrsi KR. Anarsaca as the initial Manifestation of Tuberculosis Pericarditis in a child. Journ Paed Inf Dis. 2017; 26 : 2019.

8. Bansal M, Pathak K, Pathak S. Chronic pulmonary tuberculosis and anasarca: a classic revisited. BMJ Case reports 2009; 26: 2019. PubMed: https://www.ncbi.nlm.nih.gov/pubmed/21829420

9. Airaghi I, MontoriD, Santambrogio L, Miadonna A, Tedeschi A. Chronic systemic capillary leak syndrome. Report of a case and review of the literature. Journ int med. 2001; 247: 2019. PubMed: https://www. ncbi.nlm.nih.gov/pubmed/10886496 